\title{
Enfoque educativo del software como herramienta ante la pandemia: proyecto académico QRoute
}

\author{
Educational approach to software as a tool in the face of the pandemic: QRoute \\ academic project
}

HERNÁNDEZ-CRUZ, Luz María†*, PANTÍ-GONZÁLEZ, Daniel Alberto, UICAB-BRITO, Luis Alberto y HUCHIN-POOT, Emy Guadalupe

Universidad Autónoma de Campeche. Facultad de Ingeniería. México.

Instituto Tecnológico Superior de Hopelchén, México.

ID $1^{\text {er }}$ Autor: Luz María, Hernández-Cruz / ORC ID: 0000-0002-0469-5298, Researcher ID Thomson: H-3153-2018, CVU CONACYT ID: 662220

ID $1^{\text {er }}$ Coautor: Daniel Alberto, Pantí-González / ORC ID: 0000-0001-7577-7146, Researcher ID Thomson: ABH-49852020, CVU CONACYT ID: 649888

ID $2^{\text {do }}$ Coautor: Luis Alberto, Uicab-Brito / ORC ID: 0000-0001-6986-514X, Researcher ID Thomson: ABH-5030-2020, CVU CONACYT ID: 203367

ID $3^{\text {er }}$ Coautor: Emy Guadalupe Huchin-Poot / ORC ID: 0000-0003-4430-7445, Researcher ID Thomson: ABH-49472020, CVU CONACYT ID: 538788

DOI: $10.35429 / J P D .2020 .12 .4 .19 .26$

Recibido 25 de Julio, 2020; Aceptado 30 de Diciembre, 2020

\section{Resumen}

El presente artículo despliega el análisis y evaluación del uso de Tecnologías emergentes en una solución informática para contribuir con la seguridad sanitaria ante la pandemia del COVID-19. El objetivo del estudio es vislumbrar la Tecnología con un nuevo enfoque, como una herramienta que favorezca la autonomía y el aislamiento social en beneficio de la salud. Se presenta el caso de estudio del proyecto académico QRoute, desarrollado por estudiantes universitarios que exhiben habilidades y competencias disciplinares en una solución informática integral compuesta por un sitio web y una aplicación móvil para la guía turística en Campeche. Con la aplicación del método heurístico se llevaba a cabo el análisis, evaluación y correspondencia de las Tecnologías utilizadas junto con las Estrategias principales de seguridad sanitaria impuestas a nivel nacional en México. El resultado del estudio concluye que la solución informática propuesta favorece directamente en dos Estrategias de seguridad sanitaria, el confinamiento y la sana distancia. De esta forma, se asegura que, el manejo de software puede actuar como una herramienta estratégica para la reactivación de procesos sociales, económicos y comerciales en el país.

Educación, Tecnología, Pandemia

\begin{abstract}
This article displays the analysis and evaluation of the use of Emerging Technologies in a computer solution to contribute to health security in the face of the COVID-19 pandemic. The objective of the study is to envision Technology with a new approach, as a tool that favors autonomy and social isolation for the benefit of health. The case study of the QRoute academic project is presented, developed by university students who exhibit disciplinary skills and competencies in a comprehensive computing solution composed of a website and a mobile application for the tourist guide in Campeche. With the application of the heuristic method, the analysis, evaluation and correspondence of the Technologies used together with the main health security strategies imposed at the national level in Mexico was carried out. The result of the study concludes that the proposed IT solution directly favors two health security strategies, confinement and healthy distance. In this way, it is ensured that the software management can act as a strategic tool for the reactivation of social, economic and commercial processes in the country.
\end{abstract}

Education, Technology, Pandemic

Citación: HERNÁNDEZ-CRUZ, Luz María, PANTÍ-GONZÁLEZ, Daniel Alberto, UICAB-BRITO, Luis Alberto y HUCHIN-POOT, Emy Guadalupe. Enfoque educativo del software como herramienta ante la pandemia: proyecto académico QRoute. Revista de Didáctica Práctica. 2020. 4-12:19-26.

\footnotetext{
*Correspondencia al Autor (Correo Electrónico: Imhernan@uacam.mx)
}

$\dagger$ Investigador contribuyendo como primer autor. 


\section{Introducción}

En el mundo actual nos aqueja un conjunto de condiciones adversas que han propiciado un cambio radical y abrupto en la forma en que se vive en sociedad. Al mismo tiempo, esto ha impactado en todos los ámbitos, particularmente se menciona con relación al presente estudio, el Turístico y Educativo.

Primeramente, el objetivo del estudio es vislumbrar el software como una herramienta que permita, mediante el uso de la tecnología, crear soluciones informáticas capaces de contribuir con la autonomía, aislamiento social y preservar la sana distancia en beneficio de la salud. Asimismo, como segundo punto, no menos importante, es el logro de competencias específicas (disciplinares) que permitan integrar tecnologías emergentes en el desarrollo de software para la solución de las necesidades identificadas en un proyecto particular.

En este sentido, se presenta un caso de estudio de una solución informática denominada QRoute creada por estudiantes de la Licenciatura Ingeniero en Sistemas Computacionales de la Facultad de Ingeniería de la Universidad Autónoma de Campeche, abriendo un campo de investigación aplicada de integración y aplicación tecnológica en la solución de problemas afín a la nueva normalidad.

QRoute funge como guía o asistencia turística, permite conocer la información de los sitios turísticos representativos de la Ciudad de San Francisco de Campeche, los lugares y servicios cercanos y cómo llegar a su ubicación, de forma fácil, rápida y sin la interacción física con ninguna persona.

El valor agregado de ésta, es promover la reactivación Turística en la Ciudad y Puerto de Campeche, considerando además la importancia de la misma como Patrimonio Cultural de la Humanidad en México.

Las secciones principales del artículo son: la primera sección se denomina Antecedentes, donde se expone la situación actual de México ante la pandemia del COVID19; en seguida, la segunda sección, Metodología, detalla el Proyecto QRoute, incluyendo las tecnologías utilizadas.
Por último, la tercera sección, Resultados, la cual presenta la solución informática QRoute y su análisis de correspondencia en beneficio de las Estrategias de seguridad sanitaria impuestas por el gobierno de México.

\section{Antecedentes}

El COVID-19 es la enfermedad infecciosa causada por el coronavirus que se ha descubierto recientemente y que ha invadido todo el mundo hasta convertirse en una pandemia. Una persona puede contraer la COVID-19 por contacto con otra que esté infectada por el virus. La enfermedad se propaga principalmente de persona a persona a través de las gotículas que salen despedidas de la nariz o la boca de una persona infectada al toser, estornudar o hablar. Estas gotículas son relativamente pesadas, no llegan muy lejos y caen rápidamente al suelo. Una persona puede contraer el COVID-19 si inhala las gotículas procedentes de una persona infectada por el virus. Por eso es importante mantenerse al menos a un metro de distancia de los demás. Estas gotículas pueden caer sobre los objetos y superficies que rodean a la persona de modo que otras personas pueden infectarse si tocan esos objetos o superficies y luego se tocan los ojos, la nariz o la boca. (Organización Mundial de la Salud, 2020).

En México el arribo de la pandemia estuvo marcado por un doble discurso inicial de las autoridades que, al mismo tiempo que informaban $y$ aseguraban tener ya lista la estrategia para enfrentar el desafío, por otro la parecían minimizar el impacto que la enfermedad tendría en el país (Núñez Bustillos, y otros, 2020).

El 28 de febrero de 2020 el epidemiólogo y subsecretario para la Prevención y Promoción de la Salud en la Secretaría de Salud, Hugo López Gatell confirmaba el primer caso en México. Entre otras medidas para la seguridad sanitaria generada por la emergencia ante el COVID-19 se encuentran las que refieren al cumplimiento del resguardo domiciliario y a la limitación de la movilidad voluntaria, haciendo especial énfasis a personas mayores de 60 años, mujeres embarazadas y en personas con enfermedades crónicas o autoinmunes (Gobierno de México, 2020). 
Debido a la disposición de las medidas para la seguridad sanitaria, la movilidad posible debido al turismo también fue llevada a una cancelación y suspensión de las actividades relacionadas; enfatizando que el turismo constituye un fenómeno social, cultural y económico que incluye el desplazamiento de personas a lugares fuera de su entorno habitual por diversos motivos como lo son personales, profesionales o de negocios (Organización Mundial del Turismo, OMT, 2020) y la interacción entre personas.

No obstante, en la actualidad, la situación epidemiológica de contagios y defunciones del país ha ido en "decremento" y el semáforo en la mayoría de los Estados ha permitido la reactivación de actividades económicas siguiendo los protocolos de seguridad sanitaria.

En México se trabaja con la reactivación progresiva de las actividades económicas con las medidas de seguridad sanitaria.

\section{Metodología}

La Universidad Autónoma de Campeche (UAC) es una universidad pública, autónoma, con vínculos estatales, nacionales e internacionales, que contribuye con pertinencia y calidad competitiva a la formación de capital humano y a la generación, aplicación e innovación de conocimientos para atender los requerimientos y oportunidades del desarrollo sustentable del estado de Campeche (Universidad Autónoma de Campeche, 2020).

La Facultad de Ingeniería de la UAC oferta, entre sus seis Programas Educativos, la Licenciatura en Ingeniería en Sistemas Computacionales (ISC). Programa académico acreditado por los Comités Interinstitucionales para la Evaluación de la Educación Superior (CIEES) al cual se le otorgo el Nivel 1 de cinco años, al padrón de programas de educación superior reconocidos por su Buena Calidad de los CIEES, por cumplir con los requisitos establecidos, con una vigencia de junio de 2017 a julio de 2022 (Universidad Autónoma de Campeche, 2020).
El perfil de egreso del Programa ISC es "Formar Profesionales con capacidades y habilidades individuales, capaz de desempeñarse con alto grado de compromiso social y del medio ambiente en diferentes entornos con sentido metodológico, de liderazgo y cultura emprendedora al desempeñarse analizando, diseñando, implementando, y gestionando sistemas de computación, redes y bases de datos con los estándares tecnológicos vigentes" (Universidad Autónoma de Campeche, 2020).

Los universitarios al cursar las asignaturas del núcleo integral, en los últimos dos semestres, desarrollan proyectos que permiten adquirir las competencias disciplinares del uso, aplicación, implementación e integración de diversas tecnologías en la solución de problemas y necesidades detectadas en el sector primario, industrial y/o de servicios.

Especialmente, los proyectos ostentados en el ciclo escolar 19-2, cuyo plan de estudios sufrió adecuaciones y fue adaptado a la modalidad en línea por decreto de la Secretaría de Educación Pública del país como resultado del confinamiento, han mostrado grandes oportunidades en el quehacer cotidiano y con un nuevo enfoque, resaltando la importancia de la automatización y la autonomía, gracias al uso del software.

QRoute es un proyecto de desarrollo de software que pretende sustituir las visitas guiadas de forma presencial por una aplicación móvil, con la finalidad de ofrecer una herramienta que permita reactivar el Turismo y, al mismo tiempo, preservar la salud. La Figura 1 muestra el Logotipo de QRoute.

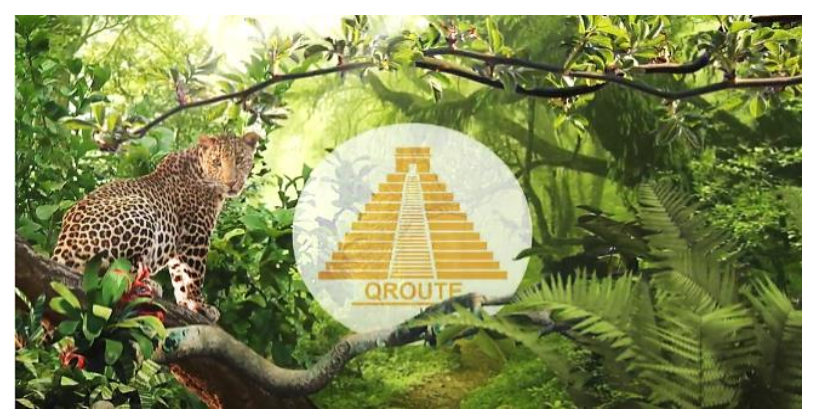

Figura 1 Logotipo QRoute

Fuente: Fuente propia 
La solución informática QRoute está compuesta por dos componentes principales: un sitio web y una aplicación móvil. El sitio web está orientado a cumplir dos propósitos importantes, primero, la administración de los clientes, quienes contratan un servicio de publicidad y que podrán ser "vistos" por los usuarios (turistas o visitantes). Y, el segundo, descargar la aplicación móvil como turista o visitante. La Figura 2 representa los componentes de QRoute y la Figura 3 muestra la solución informática QRoute.

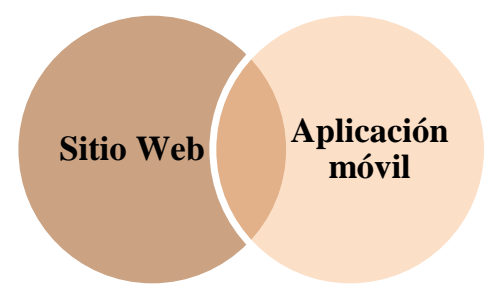

Figura 2 Componentes de la Solución Informática QRoute

Fuente: Fuente propia
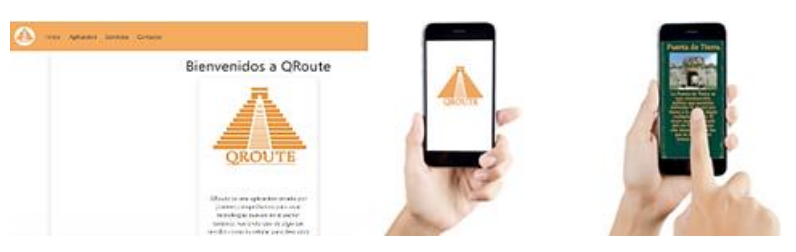

Figura 3 Componentes de la Solución Informática QRoute

Fuente: Fuente propia

Nunca se debe perder de vista, que los proyectos académicos, como QRoute, tienen la pretensión de que los estudiantes apliquen en la práctica los conocimientos adquiridos, y sean capaces de integrar las habilidades y competencias requeridas para el logro de su perfil de egreso profesional.

\section{Principales tecnologías integradas en QRoute}

El desarrollo de software no es una tarea fácil, la Ingeniería de Software es una rama de las Ciencias de la Computación que estudia la creación de software confiable y de calidad basándose en métodos y técnicas de ingeniería. La Ingeniería de Software es la aplicación de un enfoque sistemático, disciplinado y cuantificable al desarrollo, operación y mantenimiento de software.
Con lo anterior, entre otras cosas, se puede discernir claramente la importancia de integrar correctamente diferentes tecnologías emergentes que permitan optimizar la funcionalidad de una solución informática, y del mismo modo, cubrir y satisfacer los requerimientos y necesidades de los cuales emana.

Es significativo mencionar, al hablar de Tecnología, el uso del enfoque ágil en la gestión del proyecto (Hernández Cruz \& Díaz Bravo, 2020) durante su implementación, con el uso de la metodología Scrum y el empleo de la herramienta de software iceScrum (Hernández Cruz, Mex Alvarez, \& Cab Chan, 2019).

Al referirnos exclusivamente al presente estudio, el sitio web de QRoute consta de un formulario de registro para los clientes comerciales, que buscan la publicidad y el marketing. Además, la gestión del servicio que se ofrece es totalmente en línea, siendo posible llevarlo a cabo desde la cotización hasta la contratación sin requerir de la presencia física de los clientes.

La funcionalidad esencial provista por la aplicación móvil consta de tres acciones básicas: Escaneo de Código QR, despliegue de la información Turística del Código QR y la Ubicación de los lugares comerciales, restaurantes, tiendas, entre otros. La Figura 4 muestra las tres acciones básicas de la aplicación móvil QRoute.

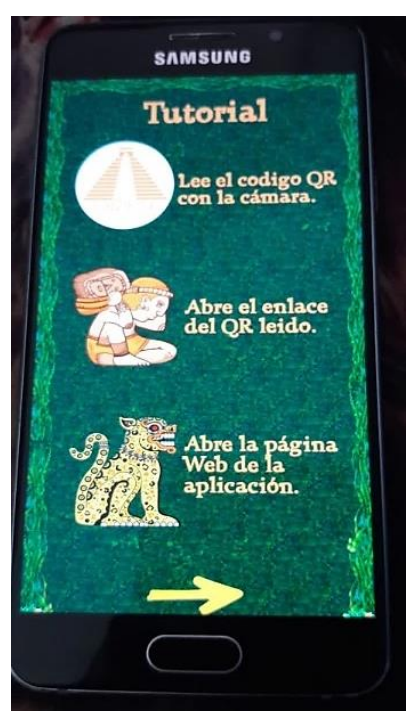

Figura 4 Acciones básicas de la Aplicación móvil QRoute

Fuente: Fuente propia 
Actualmente la aplicación solo contempla la Ciudad de Campeche, sin embargo, juntamente con la evaluación del prototipo se está redefiniendo un nuevo alcance para incluir todos los municipios del Estado.

Las Tecnologías utilizadas para implementar estas acciones se especifican a continuación.

Un Código $Q R$ (Quick Response Code Código de respuesta rápida) es un sistema para almacenar información en una matriz de puntos o un código de barras bidimensional. La facilidad de leer el código QR desde dispositivos móviles favorece múltiples aplicaciones, desde la publicidad y el marketing a la simple identificación (Allueva Pinilla \& Alejandre Marco, 2017).

Los Servicios de Google Play o Google Play Services son una aplicación que se encarga de articular múltiples elementos en cualquier dispositivo Android. Es una librería creada por Google por la cual los desarrolladores pueden aprovechar las API y servicios de Google fácilmente. Entre las que se incluye la API Vision que permite el reconocimiento de imágenes, entre ellas, los códigos de barra (barcode), y de ahí, con una constante específica, los códigos de respuesta rápida (QR) (Google Developers, 2020).

App Inventor es un producto desarrollado por el Instituto Tecnológico de Massachusetts (MIT) junto con Google, de manera que como requisito se debe disponer de una cuenta de Google para acceder a la web de MIT App Inventor. Es una herramienta web de programación visual donde se crean apps para dispositivos Android mediante la unión de bloques se dota de instrucciones a los componentes que vayan a formar tus apps, creando desde aplicaciones muy simples hasta aplicaciones mucho más complejas.
Google Maps Platform es una interfaz de programación de las APIs de Google Maps. Por medio de esta herramienta es posible implementar mapas y funciones asociadas a Google en un sitio web o aplicaciones (Svennerberg, 2010). Google Maps Platform - Maps cuenta con dos recursos: Maps y Street View. Estos permiten a los usuarios visualizar el mundo real, por medio de mapas estáticos $\mathrm{o}$ interactivos.

Como punto inicial de la investigación se resalta la importancia de los componentes de QRoute en relación con valorar las características propias de la solución informática primordialmente de acuerdo en el contexto del confinamiento y los protocolos de seguridad sanitaria.

Sitio Web QRoute. El concepto de aplicación web ofrece la oportunidad de acceso a aplicaciones a través de computadoras y otros dispositivos móviles cuando se tiene acceso a Internet. Entre las ventajas más significantes se puede mencionar que no requieren instalación (en los clientes), información centralizada y movilidad (Ferrer Martinez, 2015).

Aplicación Móvil QRoute. La principal característica que presentan los dispositivos móviles es el concepto de "movilidad", ya que son pequeños y fáciles de operar durante los traslados. Además, es posible sincronizarlos con algún sistema de computadora, para actualizar aplicaciones $y$ datos con algunas capacidades de procesamiento, con conexión permanente o esporádica a una red y con memoria limitada (Santiago \& Trabaldo, 2015).

La aplicación móvil QRoute es el componente de la solución que responde a la guía turística en la Ciudad de Campeche, México. Su uso puede expresarse en una serie de pasos que facilitan su interacción con el turista (visitante). La Figura 5 muestra el algoritmo principal de uso de la aplicación móvil QRoute. 


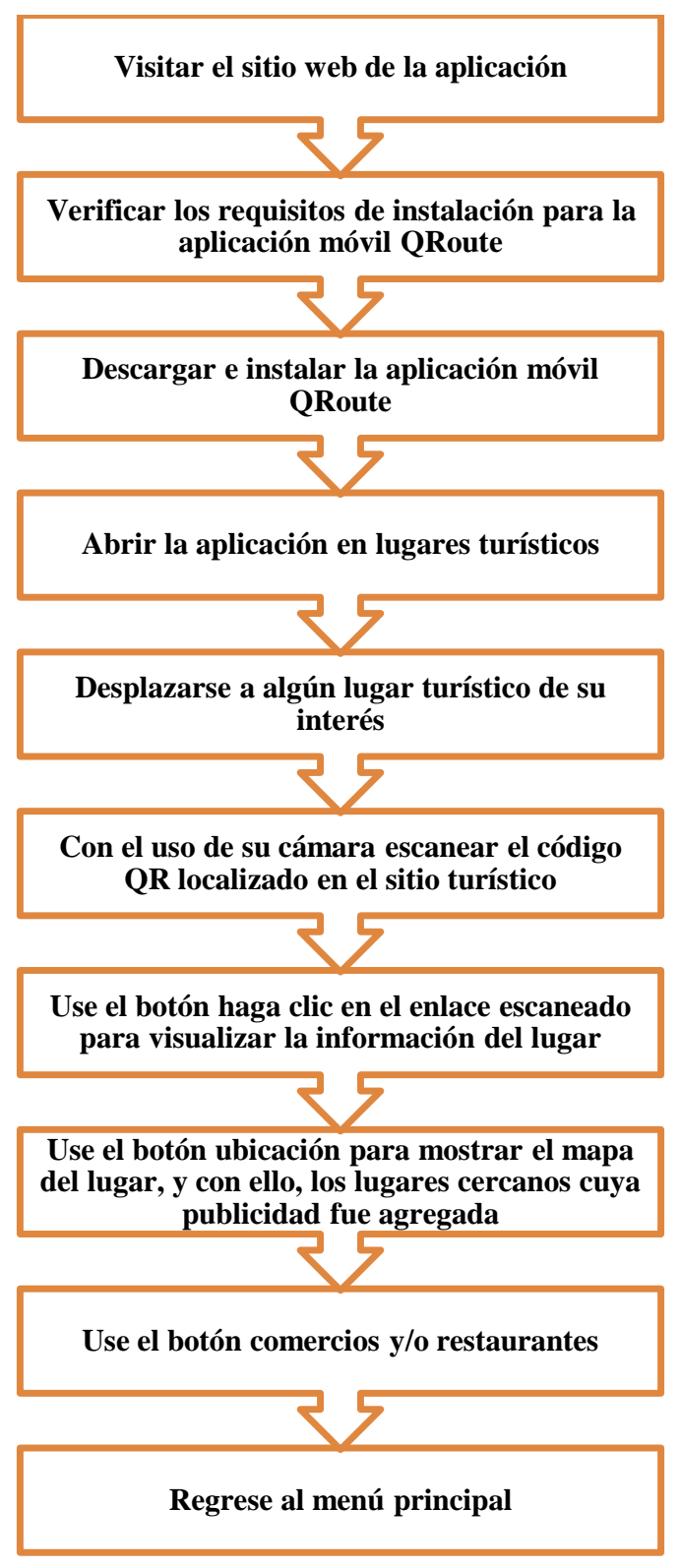

Figura 5 Proceso de uso de la aplicación móvil QRoute Fuente: Fuente propia.

\section{Resultados}

Una vez que, se ha comprendido el proceso central de la aplicación móvil QRoute, se inicia la etapa de análisis y evaluación de su uso como herramienta de guía turística en beneficio de la nueva normalidad.

La evaluación heurística es un método en el cual un grupo de expertos inspecciona un producto de software con el fin de determinar si se adhiere o no a un conjunto de principios o heurísticas de diseño. La palabra "heurística" viene de la griega "eureka". La evaluación heurística, se refiere a una serie de principios generales que consideran los expertos para llevar a cabo dicha evaluación. Este método se emplea para poder realizar la correspondencia de la solución informática con las estrategias implementadas ante la pandemia.
La Figura 6 muestra un esquema de la evaluación.

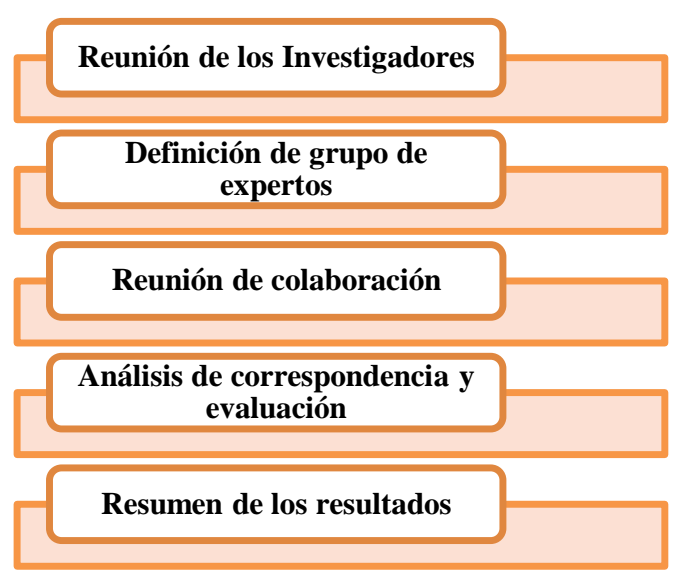

Figura 6 Proceso de Evaluación de Correspondencia como Herramienta ante la pandemia COVID-19

Fuente: Fuente propia

El grupo de expertos evalúa QRoute bajo tres propiedades básicas: forma, contenido y uso. La Tabla 1 muestra los criterios definidos con la Técnica Delphi por el grupo de expertos para evaluar las propiedades de forma, contenido y uso.

\begin{tabular}{|l|l|l|}
\hline \multicolumn{1}{|c|}{ Forma Contenido } & Uso \\
\hline Claridad & Coherencia & Facilidad \\
\hline Presentación & Propósito & Disponibilidad \\
\hline Extensión & $\begin{array}{l}\text { Pertinencia en la } \\
\text { información }\end{array}$ & Portabilidad \\
\hline Ortografía & & Interactividad \\
\hline $\begin{array}{l}\text { Tamaño y Tipo de } \\
\text { Letra (Tipografía) }\end{array}$ & & Utilidad \\
\hline & & Autonomía \\
\hline
\end{tabular}

Tabla 1 Criterios de evaluación de la solución informática QRoute

Fuente: Fuente propia.

Después de establecer los criterios para la evaluación y reconociendo las cuatro estrategias principales impuestas por el gobierno mexicano: confinamiento, sana distancia, uso de cubre bocas y lavado de manos se realiza la evaluación de la solución informática QRoute. El Gráfico 1 muestra el resumen de los resultados obtenidos para la correspondencia en el beneficio del uso de QRoute conforme a las estrategias del COVID19. La evaluación se realiza en los dos componentes de la solución informática. 


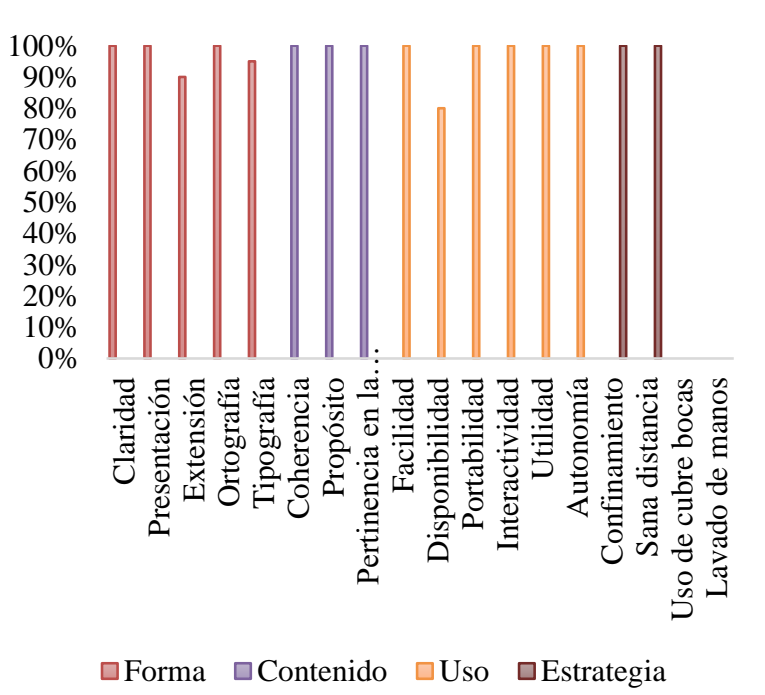

Gráfico 1 Porcentajes obtenidos por criterio en la evaluación de QRoute

Fuente: Fuente propia.

Enseguida, la Tabla 2 muestra las sugerencias y recomendaciones hechas por los expertos para los criterios que obtuvieron el menor porcentaje en la evaluación.

\begin{tabular}{|l|l|}
\hline \multicolumn{2}{|c|}{ Criterio } \\
\hline Extensión & $\begin{array}{l}\text { Contenido de información } \\
\text { demasiado resumido, considerar un } \\
\text { acervo bibliográfico actual y hacer } \\
\text { la referencia al mismo. }\end{array}$ \\
\hline $\begin{array}{l}\text { Tamaño y Tipo de } \\
\text { Letra (Tipografía) }\end{array}$ & $\begin{array}{l}\text { Aunque el tipo y tamaño de Fuente } \\
\text { es legible, por el uso de las negritas } \\
\text { podría resultar cansado a la vista. }\end{array}$ \\
\hline Uso & \multicolumn{1}{|c|}{} \\
\hline Disponibilidad & $\begin{array}{l}\text { La disponibilidad de la aplicación } \\
\text { dependerá del proveedor }\end{array}$ \\
\hline Estrategia & $\begin{array}{l}\text { El uso de la solución informática } \\
\text { QRoute no tiene ninguna relación } \\
\text { con el uso de cubre bocas. }\end{array}$ \\
\hline Uso de cubre bocas & $\begin{array}{l}\text { El uso de la solución informática } \\
\text { QRoute no tiene ninguna relación } \\
\text { con el lavado de manos. } \\
\text { De cierta forma, la recomendación } \\
\text { sería solo usar el dispositivo móvil } \\
\text { personal y no permitir que otra } \\
\text { persona pueda tomarlo físicamente. } \\
\text { Así como desinfectarlo } \\
\text { constantemente. }\end{array}$ \\
\hline Lavado de manos
\end{tabular}

Tabla 2 Recomendaciones de los criterios con menor porcentaje en la evaluación

Fuente: Fuente propia

Posteriormente la Tabla 3 muestra el resumen de los hallazgos obtenidos ante el proceso de evaluación de correspondencia del beneficio aportado a las Estrategias tomadas por el gobierno de México ante la pandemia del COVID-19 (Martín Moreno, 2020).

\begin{tabular}{|c|c|c|}
\hline $\begin{array}{l}\text { Estrategia ante el } \\
\text { COVID-19 en } \\
\text { México }\end{array}$ & $\begin{array}{l}\text { Evaluación } \\
(0-5) \\
\text { promedio de } \\
\text { correspondencia } \\
\text { del beneficio } \\
\text { obtenido }\end{array}$ & Tecnología \\
\hline E1: Confinamiento & 4 & $\begin{array}{ll}\text { Sitio Web } \\
\text { QRoute }\end{array}$ \\
\hline E2: Sana distancia & $\begin{array}{l}5 \\
4 \\
5\end{array}$ & $\begin{array}{l}\text { Aplicación } \\
\text { Móvil QRoute } \\
\text { Código QR } \\
\begin{array}{l}\text { Google Maps } \\
\text { Platform }\end{array}\end{array}$ \\
\hline $\begin{array}{l}\text { E3: Lavado de } \\
\text { manos }\end{array}$ & $\mathbf{0}$ & No Aplica \\
\hline
\end{tabular}

Tabla 3 Resumen de las Estrategias ante la pandemia y su correspondencia con las Tecnologías emergentes utilizadas en QRoute

Fuente: Fuente propia

La Figura 7 muestra el uso de Codigo QR y Google Maps en la solución informática QRoute.
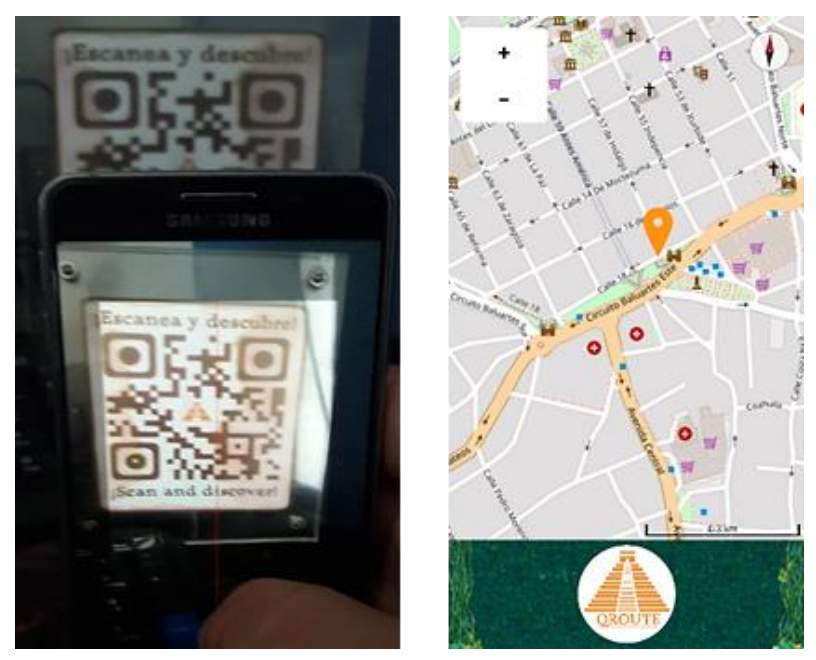

Figura 7 Manejo de Código QR y Google Maps en QRoute

Fuente: Fuente propia

\section{Agradecimiento}

Se extiende un amplio agradecimiento al Mtro. José Román Ruiz Carrillo, Rector de la Universidad Autónoma de Campeche por la disposición y apoyo brindado para la publicación del presente artículo. De igual forma, el reconocimiento al Mtro. Guadalupe Manuel Estrada Segovia, Director de la Facultad de Ingeniería y la Mtra. Nancy Georgina Ortiz Cuevas, Coordinadora del Programa Educativo Ingeniería en Sistemas Computacionales por la empatía por impulsar los estudios de investigación en el área en Ciencias de la Computación. 


\section{Conclusiones}

No cabe la menor duda que nuestro país, México, no estaba preparado para enfrentar la pandemia del COVID-19. Toda la actividad económica se ha desplomado con el confinamiento y, hoy día, tras la reactivación parcial no se ha logrado alcanzar una estabilidad, en ningún sentido. Es por esto, de suma importancia buscar alternativas estratégicas para poder adaptarnos a la nueva normalidad y encontrar otros caminos o medios que nos lleven a la productividad, redefiniendo procesos empresariales y comerciales.

Específicamente, como resultado del estudio realizado, al analizar las Estrategias de seguridad sanitaria se identifican y reconocen las herramientas tecnológicas de Código QR y API Google Maps que pueden integrarse fácilmente a las soluciones informáticas que permitan el desarrollo de software a la medida para cubrir los requisitos y necesidades de la nueva normalidad. Establecer y fortalecer mecanismos y procesos relacionados con el uso eficiente de las Tecnologías de la Información y las Comunicaciones, además de la administración, procesamiento y disponibilidad de los datos críticos son factores de éxito en la lucha contra la pandemia.

\section{Referencias}

Allueva Pinilla, A. I., \& Alejandre Marco, J. L. (2017). Aportaciones de las tecnologías como eje en el nuevo paradigma educativo. Prenzas de la Universidad de Zaragoza. Obtenido de ISBN 978-84-16933-99-0

Ferrer Martinez, J. (2015). Implantación de aplicaciones web en entornos internet, intranet y extranet. Ra-Ma.

Gobierno de México. (01 de noviembre de 2020). Gobierno de México. Obtenido de https://coronavirus.gob.mx/covid-19/

Google Developers. (2020). Google APIs for Android.

Hernández Cruz, L. M., Mex Alvarez, D., \& Cab Chan, J. R. (2019). Usabilidad de Icescrum y Srumdesk como herramientas de gestión de proyectos ágiles Scrum. 4to. Congreso Internacional de Ciencias de la Ingeniería 2 CONINCI 2019.
Hernández Cruz, L., \& Díaz Bravo, B. (2020). Diseño de un procedimiento para la gestión ágil de proyectos de desarrollo de software alineados a la guía del PMBOK. RISTI(E32), Pages: 229-241. Obtenido de ISSN: 1646-9895

Martín Moreno, F. (2020). Cuando México perdió la esperanza. ALFAGUARA.

Núñez Bustillos, J. C., Rocha Quintero, J. E., Aragón , E., Urrea Sánchez, G., Nuñez de la Peña, F. J., \& Guerrero Anaya, L. J. (2020). Covid-19 en un país de alto riesgo (Análisis Plural). Méxocp: Publicaciones de ITESO. Obtenido de ISBN 978-607-8616-92-3

Organización Mundial de la Salud. (2020). Organización Mundial de la Salud. Obtenido de https://www.who.int/es

Santiago, R., \& Trabaldo, S. (2015). Mobile learning: Nuevas realidades en el aula. Grupo Oceano.

Svennerberg, G. (2010). Beginning Google Maps API 3. Apress. Obtenido de ISBN 978-14302-2802-8

Universidad Autónoma de Campeche. (2020). Facultad de Ingeniería. Obtenido de https://fi.uacam.mx/

Universidad Autónoma de Campeche. (2020). Facultad de Ingeniería. Obtenido de https://fi.uacam.mx/view/paginas/9

Universidad Autónoma de Campeche. (2020). Universidad Autónoma de Campeche. Obtenido de https://uacam.mx/paginas/ver/7 